\title{
VARIA
}

\section{SOBRE EL DESCONOCIDO COMITENTE DE SANTO DI TITO DEL PESAR ANTE EL CRISTO MUERTO DE LA GALLERIA DELL'ACCADEMIA DE FLORENCIA: EL ESPAÑOL HERNANDO SASTRE}

\author{
Carlos Plaza
}

Universidad de Sevilla

\begin{abstract}
El artículo confirma definitivamente la identidad del comitente español de la obra de Santo di Tito del Pesar ante el Cristo muerto, construyendo también un perfil biográfico que da a conocer su importante papel político y social en la Florencia del Quinientos, esclareciendo así un poco más la realización y el encargo de la importante obra, hoy conservada en la Galleria dell'Accademia de Florencia.

Palabras clave: Pintura florentina del siglo XVI; Santi di Tito; Hernando Sastre.
\end{abstract}

\section{ON THE UNKNOWN PATRON OF SANTO DI TITO'S LAMENTATION OF CHRIST IN THE GALLERIA DELL'ACCADEMIA, FLORENCE: THE SPANIARD HERNANDO SASTRE}

This article definitively confirms the identity of the Spanish patron of Santo di Tito's painting Lamentation of Christ, and constructs a biographical profile revealing the major political and social role played by Hernando Sastre in 16th century Florence, thereby helping to further clarify why this important work, now in the Galleria dell'Accademia in Florence, was commissioned and painted.

Key words: 16th Century; Florentine painting; Santi di Tito; Hernando Sastre.

En 1934 Günther Arnolds reconoce Il Compianto davanti al Cristo morto como obra del pintor Santi di Tito gracias a la firma "SANCTI TITIUS" presente en la pintura, la cual se encontraba en aquel tiempo en los depósitos de la Galleria dell'Accademia de Florencia y atribuida al taller del pintor de Borgo San Sepolcro (fig. 1). El estudioso evidencia el hecho de que los figurantes se encuentren representados en la obra, con la única excepción de la Virgen, como pastores adorando a Cristo. A San Juan Bautista y Santa Catalina de Siena es necesario añadir al comitente, un desconocido caballero de Santo Stefano que añade, según el estudioso, un carácter excepcional a la composición iconográfica de esta piedad ${ }^{1}$. Precedentemente, la obra no es mencionada en la

\footnotetext{
${ }^{1}$ Arnolds, 1934: 41-42.
} 




Fig. 1. Santo di Tito, Pesar ante el Cristo muerto, 1576-87, Firenze, Galleria dell'Accademia.

guía de la pinacoteca de 1884 entre aquéllas expuestas en el museo², pero después de la identificación de Arnolds la guía oficial de 1936 publicó la obra Cristo deposto dalla Croce "con la vergine, S. Giovanni Battista, S. Caterina d'Alessandria e un committente" como obra de Santi di Tito, citando además como la misma "pervenne nelle Gallerie dalla cappella della Fortezza da Basso di Firenze nell'agosto del $1865^{\prime \prime 3}$, tal y como se observa en el llamado Inventario $1890^{4}$.

${ }^{2}$ Pieraccini, 1884.

3 Procacci, 1936: 47

${ }^{4}$ Polo Museale Fiorentino, Inventario Generale dei Dipinti. Inventario 1890, nº. 4637, reg. 6: 196-197, "Descrizione del soggetto: Il Deposto, Il Cristo morto giace in terra, [...] più innanzi un cavaliere in armatura colla croce di S. Stefano sul petto pure in ginocchio. Presso di lui uno stemma con un pino, una rota e la croce rossa in un campo bianco traversato da una fascia rossa. Particolarità storico artistiche: Estratto della cappella della Fortezza da Basso di Firenze, Agosto 1865”.

Arch. esp. arte, LXXXVIII, 349, ENERO-MARZO 2015, 85-92 ISSN: 0004-0428, eISSN: 1988-8511, doi: 10.3989/aearte.2015.06 
Sucesivamente, Jack Spalding, en su importante monografía del artista de 1982, realiza la hipótesis que el comitente representado fuese el español Baltasar Suárez de la Concha. Esta identificación se apoyaba en la relación clientelar existente entre ellos al ser mencionado por Filippo Baldinucci el encargo de Baltasar a Santi di Tito de un fresco en el claustro grande de Santa María Novella ${ }^{5}$, lo que induce al estudioso a datar esta obra en el mismo período que el fresco del convento, es decir, en los años Setenta del siglo $\mathrm{XVI}^{6}$. Esta hipótesis no es aceptada por la crítica, y en la guía de 1999 se declara como no ha sido posible reconocer al comitente ${ }^{7}$. Recientemente, el personaje ha sido identificado como el español y caballero de Santo Stefano Hernando Sastre por parte de Francesca Fumi, habiendo sido dado a conocer el hallazgo por Nadia Bastogi en un breve estudio de la obra con motivo de su última restauración, y en el cual, sobre la base de precedentes estudios, es datada la obra en $1592^{8}$.

La identificación derivaría de la individualización de las armas presentes en el escudo que aparece en la pintura y con respecto a ello la consulta de las armas de los caballeros es-

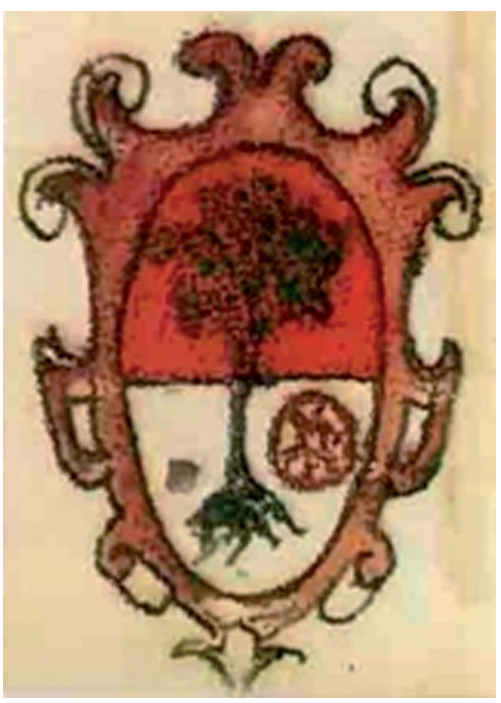

Fig. 2. Blasón Sastri, Archivio di Stato di Pisa, Ordine di Santo Stefano, f. 626.22 (de Casini, 1996: fig. 30). pañoles que pertenecieron a la Orden durante el siglo XVI, y que ellos mismos entregaron en el momento de entrar en la Orden, no admite dudas sobre la identidad del personaje: se trata del español Hernando Sastre, quien tomó el hábito el 26 de noviembre de 1576, sirviendo esta fecha como terminus post quem para la datación de la obra, como ya propuesto por Nadia Bastogi (fig. 2) ${ }^{9}$.

No obstante la definitiva identificación del personaje en una escueta ficha sobre la restauración de la obra, el español Hernando Sastre es completamente desconocido para la crítica y la historiografía florentina ${ }^{10}$. El objetivo de este breve estudio es intentar esclarecer su figura, trazando algunas notas biográficas para la construcción de un perfil aún desconocido más allá de su identidad y contextualizándolo a su vez dentro de la sociedad florentina de la segunda mitad del siglo XVI, donde el relevante papel del español es también analizado a continuación. Se afrontan igualmente cuestiones que podrían esclarecer las circunstancias hoy desconocidas entorno a la comisión de la pintura y la relación de clientela entre el pintor y el comitente de esta importante obra de la cultura pictórica de la Florencia de la segunda mitad del siglo XVI.

Carlo Sebregondi, estudioso de las familias florentinas, anota las siguientes noticias en sus apuntes sobre la familia "Sastri" de la que Hernando sería el fundador en Florencia: Ernando de D. Antonio fue "Cittadino Fiorentino, 1563. Fondatore del Priorato di Lucca, 1576. Cavaliere di

\footnotetext{
${ }^{5}$ Baldinucci, 1845-1647, II: 68.

${ }^{6}$ Spalding, 1982: 328.

Falletti, 1999: 73.

${ }^{8}$ Bastogi, 2003: 241. La datación proviene de Lecchini Giovannoni, 1984: 27-28.

9 Aún si no es mencionado en el estudio, la publicación de las armas y los datos de ingreso de todos los caballeros españoles de Santo Stefano en el siglo XVI, incluidos los de Hernando Sastre, en Casini, 1996, y revisados en CeballosEscalera Gila, Cercós García, 2007: 263-301.

${ }^{10} \mathrm{Su}$ importancia como comitente y como parte de la comunidad de españoles asentada en Florencia es dada a conocer brevemente por el autor de este estudio en Plaza, 2012: 1544. Ninguna mención en González Talavera 2012a, habiéndose retomado la noticia posteriormente en González Talavera, 2012b: 208-210.
}

Arch. esp. arte, LXXXVIII, 349, ENERO-MARZO 2015, 85-92

ISSN: 0004-0428, eISSN: 1988-8511, doi: 10.3989/aearte.2015.06 
Sto. Stefano, 1576. Castellano della fortezza da Basso di Firenze. Generale di tutte le fanterie di Toscana" ${ }^{11}$. Como primera noticia sobre el personaje, encontramos a "Ernando Sastri cameriere", junto a otros españoles y con un sueldo de 192 escudos anuales, entre los asalariados de la corte ducal de $1550^{12}$. Su papel de camarero de Cosimo I es confirmado en una carta enviada por el Duque a Carlos V, ya que fue el encargado de llevar la noticia al Emperador en 1554 de la victoria sobre Siena, mencionando el Duque en dicha misiva como Hernando, a quien había criado en Florencia desde pequeño, era su camarero y se había distinguido como valiente guerrero en la toma de la ciudad ${ }^{13}$, siendo confirmado todo ello en el diario de guerra sobre la campaña de Siena escrito por el también español Antonio Ramírez de Montalvo ${ }^{14}$. Tras este viaje permanecerá en la corte imperial tal y como nos indica el "registro dei salariati" de la corte florentina de 1554, en la que Hernando recibe aún el salario aunque si está "al presente nell'Alemagna alla Corte Cesarea alla guerra" ${ }^{\prime 15}$, una corte imperial que conocería bien ya que en ella se encuentra como enviado medíceo también en 1552, tal y como sabemos a partir de una carta del español al Duque de esa fecha ${ }^{16}$. En la guerra de Siena, sabemos que estaba al mando de una "compagnia di spagnuoli" ${ }^{17}$ y el historiador Giovan Battista Adriani confirma su relevante papel en el conflicto mencionándolo como un alto militar al frente de un pequeño ejército bajo las órdenes del marqués de Marignano ${ }^{18}$. Tras la guerra tenemos en cambio pocas noticias sobre su actividad florentina: en 1559, a través de la habitual práctica cosimiana de donar bienes y créditos pertenecientes a opositores y fuoriusciti a favor de personajes de confianza, le son donados créditos pertenecientes a Bartolommeo Cavalcanti por valor de 6109 escudos, siendo mencionado en el privilegio emanado para ello por Cosimo I como "insigni peditum prefecto, et Cubiculario nostro" y elogiando en él sus virtudes ${ }^{19}$. En 1574, según el cronista florentino Giuliano de’Ricci, el español se encontraba al mando de la guarnición de la fortaleza da Basso como castellano mayor ${ }^{20}$, y dos años más tarde, en julio de 1576, tomará el hábito de caballero de Santo Stefano de manos del propio granduque Francesco I como uno de tantos españoles que tomaron el hábito durante el primer siglo de vida de la Orden ${ }^{21}$. Será precisamente su relación con la Orden Militar creada por Cosimo I lo que nos permitirá conocer su actividad durante su último período florentino. Tras su ingreso funda una commenda di patronato en la forma del Priorato di Lucca, vinculando a ella las donaciones que había recibido por parte del Duque y que pertenecieron al fuoriuscito Bartolommeo Cavalcanti, entre las cuales se encontraban créditos, casas y tierras en Florencia y Toscana ${ }^{22}$. A partir de la documentación conservada sobre él como caballero, conocemos la fecha de sus exequias celebradas en noviembre de $1587^{23}$. Tras su muerte le sucedió su hijo Marzio como prior

${ }^{11}$ Famiglia Sastri (de Sastre), ASF, Raccolta Sebregondi, leg. 4784.

${ }_{12}$ Cariche d'onore concesse dai Ser.mi G.G. Duchi. Tomo Secondo che contiene gli arrolati della corte, ASF, Manoscritti, 321, ff. 43r-44v.

${ }^{13}$ Carta de Cosimo I a Carlos V, 2 de agosto de 1554, ASF, Mediceo del Principato, leg. 324, f. 51, transcrita en SPINI, 1940: 152-153.

${ }^{14}$ Montalvo, 1863: 111-113.

15 ASF, Manoscritti, leg. 321, ff. 101r-105v.

${ }^{16}$ Carta de Hernando Sastre a Cosimo I, ASF, Mediceo del principato, leg. 410, f. 926, 27 de agosto de 1552 (BIA, Doc ID\#21297).

${ }^{17}$ Carta de Gian Luigi (Chiappino) Vitelli a Cosimo I, ASF, Mediceo del Principato, leg. 648, f. 92 (BIA, Doc ID\#22312), de 26 de noviembre de 1554.

${ }_{18}$ Adriani, 1822-1823: V, 284, confirmado por el amplio estudio de Cantagalli, 1962: 250, 518.

19 ASF, Pratica Segreta, leg. 186, s.n.

${ }^{20}$ Ricci, 1972: 141.

${ }^{21}$ Casini, 1996: 151.

${ }^{22}$ Un estudio jurídico de la encomienda en Barsanti, 1996: 191-196.

${ }^{23}$ Casini, 1996: 151. 
de Lucca, quien había sido investido caballero en noviembre de 1579 a la edad de 16 años, muriendo menos de dos años después, ya que sabemos cómo sus exequias fueron celebradas el 18 de abril de $1589^{24}$.

A la luz de estas noticias podemos reconstruir un somero perfil biográfico de Hernando Sastre así como su papel en la sociedad y en la política florentina de la época. Su llegada sería difícil de precisar, seguramente entre el inicio de la construcción de una pequeña corte tras la llegada de Leonor de Toledo en el 1539 y los numerosos asentamientos de personajes españoles en la corte florentina durante los años Cuarenta provenientes de la corte del virrey Pedro de Toledo, pero también de la de su hermano el cardenal Juan Álvarez de Toledo. En cualquier caso ha de ubicarse necesariamente dentro de la notable presencia de personajes españoles, pertenecientes a diferentes grupos sociales, en la sociedad florentina de la segunda mitad del siglo, desde militares hasta cortesanos, universitarios, religiosos o caballeros de Santo Stefano. Las motivaciones y características fundamentales de este extraordinario y poco conocido asentamiento español en la ciudad toscana han sido analizadas por el autor del presente estudio como premisa para el estudio de interesantes encargos arquitectónicos realizados por parte de algunos de sus exponentes más importantes ${ }^{25}$.

Llegado quizás como paje, o con un papel menor en la corte, sería por Cosimo "allevato da me da piciol fanciulo", como él mismo escribe a Carlos V. Tras la entrega de las fortalezas por parte del Emperador a Cosimo I en 1543, la custodia de las mismas fue a su vez confiada por Cosimo a los soldados españoles ${ }^{26}$, quienes sucesivamente serán también protagonistas en la creación del nuevo ejército florentino que comenzaba a organizar el Duque sobre el modelo español y sobre el cual se conoce muy poco a día de hoy ${ }^{27}$. No obstante es Scipione Ammirato quien menciona que fue confiado a los militares españoles un papel protagonista en la formación del nuevo ejército toscano debido a la desconfianza del Duque para con sus connacionales ${ }^{28}$, e incluso dos siglos más tarde el escritor y editor Gaetano Cambiagi menciona la creación por Cosimo I en 1544 de una guardia personal formada por sesenta coraceros españoles ${ }^{29}$.

Por lo tanto, el joven Sastre habría pasado de la carrera áulica a dirigir su carrera hacia las armas, adquiriendo protagonismo en la reorganización de la milicia bajo Cosimo I; sobre ello, desconocemos fuentes quinientistas que nos confirmen o desmientan el papel de "generale di tutte le fanterie di Toscana" que menciona Carlo Sebregondi. Con la llegada de la guerra de Siena, el ya investido capitán Sastre tomaría parte muy activa en ella al mando de una compañía y tras haber destacado en la batalla sería recompensado por Cosimo con el honor de llevar la noticia de la victoria al Emperador y a su hijo. Tras la guerra y su estancia en la corte imperial, el capitán Sastre permanecerá en Florencia como castellano mayor de la fortaleza da Basso, de acuerdo con la po-

\footnotetext{
24 Idem: 154.
}

25 Plaza, en prensa, cfr. PlazA, 2012 y 2013. En 2012 es publicada la tesis doctoral de Blanca González Talavera, González Talavera, 2012b. Dicho estudio es interesante y novedoso en lo que respecta al análisis de conjunto de algunos ejemplos de committenza pittorica spagnola, fundamentalmente capillas gentilicias presentes en iglesias y conventos de la ciudad. En cambio, el análisis y las consiguientes consideraciones sobre la importancia política de los españoles en el nuevo estado recoge consideraciones ya puestas en evidencia por el autor del presente estudio en 2010 en una comunicación en el XVIII Congreso Español de Historia del Arte. Mirando a Clío. El arte español reflejo de su historia, Universidad de Santiago de Compostela, 20-24 de septiembre de 2010, publicada posteriormente en Plaza, 2012, y anticipándose gran parte de los contenidos en el libro de resúmenes de la citada reunión científica. Ninguna novedad añade la misma autora en un estudio publicado recientemente, González Talavera, 2013.

26 Sobre la cuestión de las fortalezas en manos de Carlos V, véase Spini, 1945: passim, y sobre la guarnición española de la fortaleza, véase Plaza, 2011 y Plaza, 2013: 67-80.

27 El estudio más importante es aún Giorgetti, 1916; véase también Ferretti, 1929-1930.

28 Ammirato, 1846: VI, 268, 363.

${ }^{29}$ Cambiagi, 1766: 126, 135. Agradezco la señalación de esta referencia bibliográfica a Maurizio Arfaioli.

Arch. esp. arte, LXXXVIII, 349, ENERO-MARZO 2015, 85-92

ISSN: 0004-0428, eISSN: 1988-8511, doi: 10.3989/aearte.2015.06 
lítica de Cosimo de confiar su guardia a personajes españoles, como es puesto en evidencia por los embajadores de Venecia en Florencia Lorenzo Priuli y Andrea Gussoni en sus respectivas relaciones al Senado de 1566 y $1576^{30}$.

Gracias a la carrera de Hernando al servicio de Cosimo I el granduque Francesco le concedió el hábito de Santo Stefano en 1576 con el privilegio de no tener que presentar pruebas de nobleza, certificando el mismo Granduque sus méritos para ser investido como caballero, algo poco habitual si consultamos los fascículos coetáneos de las pruebas de ingreso en la Orden. Dichos méritos habrían hecho que Cosimo le donase, algunos años antes, bienes confiscados a personajes florentinos con los cuales era habitual que el Duque recompensara a sus más fieles colaboradores en una práctica de economía política bien conocida en el régimen y que ha sido definida como patrimonialism $^{31}$. Los mismos bienes serían vinculados por el español a la encomienda que Francesco I le concedió fundar ese mismo año: el Priorado de Lucca. Come hemos visto, a su muerte en 1587 le sucedió su hijo Marzio quien muere apenas dos años después que su padre, en 1589, extinguiéndose así la familia en Florencia.

El capitán Hernando Sastre perteneció así a la categoría social de los "uomini nuovi” a través de los cuales Cosimo procedió a la construcción del aparato burocrático, político, áulico, social y militar del principado creando un nuevo patriciado toscano, usando como fieles colaboradores a personajes pertenecientes a la pequeña nobleza florentina y toscana, pero sirviéndose también de un importante número de personajes españoles ${ }^{32}$. Mientras otros personajes fueron eficaces burócratas -como Ugolino Grifoni de San Miniato, Pietro e Lorenzo Usimbardi de Colle Val d'Elsa y Bartolommeo Concino de Terranuova-, o leales cortesanos -como Sforza Almeni de Perugia, Stefano Alli de Roma o Leonardo Marinozzi pero también, y con un papel muy destacado en la corte florentina, los españoles Antonio Ramírez de Montalvo, Fabio Arrazola de Mondragón, Antonio Vega o Baltasar Suárez de la Concha-, otros, en cambio, fueron personajes que debieron su éxito social al fiel ejercicio de las armas bajo el mando de Cosimo, pudiendo mencionarse a los aretinos Otto y Federigo da Montauto, el almirante Jacopo Inghirami de Volterra y, proveniente también de una tradición familiar filo medícea, a Chiappino Vitelli. Es en esta categoría donde es preciso ubicar a Hernando Sastre; un personaje que alcanza una posición relevante en la Florencia del período pero que, a diferencia de otros personajes españoles que adquieren notoriedad en coetáneas carreras dentro del granducado, su memoria desaparecerá tras la muerte de su hijo como último representante de la familia apenas dos años después de su propia muerte.

Este personaje, como hemos visto, ocupaba un papel importante en la ordenación militar de Cosimo y Francesco al mando de las guarniciones españolas de las fortalezas, explicando esto el hecho de que la obra del Pesar ante el Cristo muerto, encargado entonces entre 1576 y $1587^{33}$, se

${ }^{30}$ Lorenzo Priuli en su relación de 1566 informa: "Per mantenersi [il Duca Cosimo] la grazia del re [Filippo II], mostra di stimare e fidarsi nella nazione spagnuola per guardia delle sue fortezze di Fiorenza e di Livorno". Andrea Gussoni en 1576 informa: "Tiene il granduca [Francesco I] in San Miniato per guardia quaranta Spagnuoli, e nel Castello [Fortezza da Basso] cento della medesima nazione, e fa questo il principe come cosa principata dal padre, che voleva con questo mezzo gratificare quella nazione, dimostrando confidenza in essa"; Alberi, 1839-1863: II.II, 81. Esta noticia se confirma con otros españoles al mando de las fortalezas florentinas, véase Plaza, 2013: 67-80.

${ }^{31}$ Litchfield, 1986: 157. La donación ducal como gratificación a los colaboradores medíceos es analizado también en Fantoni, 1994: 97-122.

32 Sobre ellos véase Plaza, 2013: 95-105.

${ }^{33}$ Fechas de ingreso en la Orden y de muerte de Hernando Sastre. Sería menos probable que la obra hubiese sido encargada por Marzio en honor de su padre, lo que desplazaría el encargo a 1589.

Arch. esp. arte, LXXXVIII, 349, ENERO-MARZO 2015, 85-92 ISSN: 0004-0428, eISSN: 1988-8511, doi: 10.3989/aearte.2015.06 
encontrase en la desaparecida capilla de la fortaleza de San Giovanni o da Basso, posiblemente en uno de sus tres altares ${ }^{34}$, antes de ser trasladada a la colección de la Galleria dell'Accademia de Florencia en 1865.

\section{BIBLIOGRAFÍA}

Adriani, Giovan Battista (1822-1823): Istoria de' suoi tempi, (ed. de Adriani, Marcello), I-VIII, Prato, Giacchetti.

Alberi, Eugenio (ed.) (1839-1863): Relazioni degli ambasciatori veneti al Senato, I-XV, Florencia, Società Editrice Fiorentina.

Ammirato, Scipione (1846): Istorie fiorentine di Scipione Ammirato. Con l'aggiunte di Scipione Ammirato il giovane (ed. de Ranalli, Ferdinando), I-VI, Florencia, Batelli e Compagni.

Arnolds, Günther (1934): Santi di Tito. Pittore di Sansepolcro, Arezzo, R. Accademia Petrarca.

Baldinucci, Filippo (1845-1847): Notizie de'professori del disegno da Cimabue in qua, Firenze 1681-1728 (ed. de Ranalli, Ferdinando, reed. Florencia, 1974).

Barsanti, Danilo (1996): "Presenze spagnole a Firenze nel sec. XVI: le commende di patronato Sastri e Suarez nell'Ordine di Santo Stefano". En Toscana e Spagna nel secolo XVI. Miscellanea di studi storici, Pisa, ETS, pp. 191-196.

Bastogli, Nadia (2003): "Compianto di Cristo con San Giovanni Battista, Santa Caterina d'Alessandria e il donatore". En Falletti, Franca, Scuderi, Magnolia, (eds.), Intorno al David. La grande pittura del secolo di Michelangelo, Florencia, Giunti.

Cambiagi, Gaetano (1766): Memorie storiche riguardanti le feste solite farsi in Firenze per la natività di $S$. Gio. Battista, Protettore della Città e dominio fiorentino, Florencia, Stamperia Granducale.

Cantagalli, Roberto (1962): La guerra di Siena 1552-1559. I termini della questione senese nella lotta tra Francia e Asburgo nel '500 e il suo risolversi nell'ambito del Principato Mediceo, Siena, Accademia Senese degli Intronati.

Casini, Bruno (1996): "I cavalieri spagnoli membri del Sacro Militare Ordine di Santo Stefano nel secolo XVI". En Toscana e Spagna nel secolo XVI. Miscellanea di studi storici, Pisa, ETS, pp. 123-189.

Ceballos-Escalera Gila, A., Cercós García, L.F. (2007): "Españoles en la orden de San Esteban de Toscana: caballeros pretendientes y falsarios". En M. Aglietti (ed.), Istituzioni, potere e società: le relazioni tra Spagna e Toscana per una storia mediterranea dell'Ordine dei Cavalieri di Santo Stefano, "Quaderni Stefaniani", XXVI, pp. 263-301.

Falletti, Franca (1999): Guida ufficiale, Firenze, Giunti.

Fantoni, Marcello (1994): La corte del granduca. Forme e simboli del potere mediceo tra Cinque e Seicento, Roma, Bulzoni Editore.

Ferretti, Jolanda (1930): L'organizzazione militare in Toscana durante il governo di Alessandro e di Cosimo I de’Medici. En "Rivista Storica degli Archivi Toscani”, I, 1929, pp. 248-276; II, pp. 58-80.

Giorgetti, Niccolò (1916): Le armi toscane e le occupazioni straniere in Toscana (1537-1860), I-III, Città di Castello, Tip. Unione Arti Grafiche.

González Talavera, Blanca (2012a): "La comunidad española de la Florencia medícea (1539-1600): principales manifestaciones artísticas". En Mirando a Clio: el arte español espejo de su historia. Actas del XVIII Congreso Español de Historia del Arte (Facultad de Geografía e Historia, Universidad di Santiago

${ }^{34}$ Sobre la iglesia o capilla de la fortaleza de San Giovanni se conoce poco. En un inventario de finales del siglo XIX es mencionada "una chiesa a tre navate, che due a tetto e quella di mezzo stojata, tre altari, e balaustri di legno e quello di mezzo; sulle sinistra ci resta il Corridore, la Sagrestia, la stanza mortuoria e la scaletta di pietra per ascensore alla Cantoria, e un piccolo stanzino ove corrispondere la fusi delle campane", ASF, Fabbriche Granducali, leg. 2815, transcrito en Gurrieri, Manzoni, 1990: 169-170.

Arch. esp. arte, LXXXVIII, 349, ENERO-MARZO 2015, 85-92

ISSN: 0004-0428, eISSN: 1988-8511, doi: 10.3989/aearte.2015.06 
de Compostela, 20-24 septiembre, 2010); Barral Rivadulla, María Dolores, et al. (eds.), Santiago de Compostela, pp. 1262-1263.

González Talavera, Blanca (2012b): Presencia y mecenazgo español en la Florencia medicea: de Cosme I a Fernando I, tesis doctoral, Universidad de Granada, Università degli Studi di Firenze, codirectores Antonio J. Calvo Castellón, Mara Visonà.

González Talavera, Blanca (2013): "Presencia y mecenazgo español en la Florencia medícea del Quinientos". En Anales de Historia del Arte, XXIII, pp. 395-406.

Gurrieri, Francesco; Manzoni, Paolo (1990): La Fortezza da Basso: un monumento per la città, Florencia, Ponte alle Grazie.

Lecchini Giovannoni, Simona (1984): Studi e disegni preparatori di Santi di Tito. En "Paragone", VL, pp. 20-36.

Litchfield, Richard Burr (1986): Emergency of a Bureaucracy. The florentine patricians 1530-1790, Princeton, Princeton University Press.

Montalvo, Antonio (1863): Relazione della Guerra di Siena (ed. de Riccomani, Cesare; Grottanelli, Francesco), Turín, Tip. Vercellino.

Pieraccini, Eugenio (1883): Guida della R. Galleria antica e moderna e tribuna del David, Florencia, Tip. Cooperativa Editrice.

Plaza, Carlos (2011): "Arquitectura militar en Italia en el siglo XVI y la aportación española: el caso de Florencia y Siena”. En Actas del VII congreso de Historia de la Construcción (Universidad de Santiago de Compostela, 26-29 de octubre de 2011); Huerta, Santiago; et al. (eds.), Madrid, Instituto Juan de Herrera, II, pp. 1133-1146.

Plaza, Carlos (2012): "Arquitectura y mecenazgo artístico de la comunidad española en la Florencia del Quinientos". En Mirando a Clio: el arte español espejo de su historia. Actas del XVIII Congreso Español de Historia del Arte (Facultad de Geografía e Historia, Universidad di Santiago de Compostela, 20-24 septiembre, 2010); Barral Rivadulla, María Dolores, et al. (eds.), Santiago de Compostela, pp. 1538-1551.

Plaza, Carlos (2013): Arquitectura, comitentes y presencia española en Florencia en el siglo XVI, tesis doctoral inédita, Universidad Hispalense, Università degli Studi di Firenze, codirectores Amedeo Belluzzi, Ana Marín Fidalgo, defendida el 20 de junio de 2013 en la Escuela Técnica Superior de Arquitectura de Sevilla.

Plaza, Carlos (en prensa): Arquitectura en Florencia y comitentes españoles en el siglo XVI, Madrid, Centro de Estudios Europa Hispánica.

Procacci, Ugo (1936): La R. Galleria dell'Accademia di Firenze, Roma, Libreria dello Stato.

Ricci, Giuliano de' (1972): Cronaca (1532-1606), (ed. de Sapori, Giuliana), Milán, Riccardi.

Sebregondi, Carlo (1940): Famiglie patrizie fiorentine, Firenze, C. Cya.

Spalding, Jack (1982): Santi di Tito, New York \& London, Garland.

Spini, Giorgio, (ed.) (1940): Lettere di Cosimo I, Florencia, Vallecchi.

Spini, Giorgio (1945): Cosimo I de'Medici e l'indipendenza del principato mediceo, Firenze, Vallecchi, (reed. 1980).

Abreviaturas:

ASF. Archivio di Stato di Firenze.

ASPi. Archivio di Stato di Pisa.

BIA, Doc ID\#. BIA: The Medici Archive Project, Doc ID\#.

Los documentos conocidos a través del Medici Archive Project son citados explícitamente (BIA: The Medici Archive Project, Doc ID\#).

Fecha de recepción: 09-XII-2013

Fecha de aceptación: 16-VI-2014

Arch. esp. arte, LXXXVIII, 349, ENERO-MARZO 2015, 85-92 ISSN: 0004-0428, eISSN: 1988-8511, doi: 10.3989/aearte.2015.06 